\title{
The Three Hundred Project: The stellar angular momentum evolution of cluster galaxies
}

\author{
R. Mostoghiu ${ }^{1,3,4} \oplus$, A. Knebe ${ }^{1,2,3}$, F. R. Pearce ${ }^{4}$, C. Power ${ }^{3,5}$, C. D. P. Lagos ${ }^{3,5}$, W. Cui ${ }^{6}$, S. Borgani ${ }^{7,8,9,10}$, \\ K. Dolag ${ }^{11,12}$, G. Murante ${ }^{8}$, and G. Yepes ${ }^{1,2}$ \\ ${ }^{1}$ Departamento de Física Teórica, Módulo 15, Facultad de Ciencias, Universidad Autónoma de Madrid, 28049 Madrid, Spain \\ e-mail: robert.mostoghiu@uam.es \\ 2 Centro de Investigación Avanzada en Física Fundamental (CIAFF), Facultad de Ciencias, Universidad Autónoma de Madrid, \\ 28049 Madrid, Spain \\ 3 International Centre for Radio Astronomy Research, University of Western Australia, 35 Stirling Highway, Crawley, \\ Western Australia 6009, Australia \\ 4 School of Physics \& Astronomy, University of Nottingham, Nottingham NG7 2RD, UK \\ 5 ARC Centre of Excellence for All Sky Astrophysics in 3 Dimensions (ASTRO 3D), Australia \\ ${ }^{6}$ Institute for Astronomy, University of Edinburgh, Royal Observatory, Edinburgh EH9 3HJ, UK \\ 7 Dipartimento di Fisica, Sezione di Astronomia, Università di Trieste, Via Tiepolo 11, 34143 Trieste, Italy \\ 8 INAF - Osservatorio Astronomico Trieste, Via Tiepolo 11, 34123 Trieste, Italy \\ 9 Institute of Fundamental Physics of the Universe, Via Beirut 2, 34151 Grignano, Trieste, Italy \\ 10 INFN, Instituto Nazionale di Fisica Nucleare, Trieste, Italy \\ 11 University Observatory Munich, Scheinerstraße 1, 81679 Munich, Germany \\ 12 Max-Planck-Institut fur Astrophysik (MPA), Karl-Schwarzschild Straße 1, 85748 Garching bei München, Germany
}

Received 15 May 2020 / Accepted 3 May 2021

\section{ABSTRACT}

\begin{abstract}
Using 324 numerically modelled galaxy clusters as provided by THE THREE HUNDRED project, we study the evolution of the kinematic properties of the stellar component of haloes on first infall. We selected objects with $M_{\text {star }}>5 \times 10^{10} h^{-1} M_{\odot}$ within $3 R_{200}$ of the main cluster halo at $z=0$ and followed their progenitors. We find that although haloes are stripped of their dark matter and gas after entering the main cluster halo, there is practically no change in their stellar kinematics. For the vast majority of our 'galaxies' - defined as the central stellar component found within the haloes that form our sample - their kinematic properties, as described by the fraction of ordered rotation, and their position in the specific stellar angular momentum-stellar mass plane $j_{\text {star }}-M_{\text {star }}$ are mostly unchanged by the influence of the central host cluster. However, for a small number of infalling galaxies, stellar mergers and encounters with remnant stellar cores close to the centre of the main cluster, particularly during pericentre passage, are able to spin up their stellar component by $z=0$.
\end{abstract}

Key words. methods: numerical - galaxies: clusters: general - galaxies: evolution - galaxies: kinematics and dynamics

\section{Introduction}

In a hierarchical model of structure formation, the structures observed today are a result of the merging of dark matter clumps at high redshift via gravitational collapse. As the clumped dark matter grows into larger objects to form haloes, they experience tidal torques from neighbouring objects. At the same time, baryons condense in the centre of such structures to form galaxies (Peebles 1969; Doroshkevich 1970; White \& Rees 1978; White 1984). During their mutual formation, both the dark matter and baryonic component experience the same tidal fields, and hence it is expected that they gain the same amount of specific angular momentum. Furthermore, considering that baryons evolve inside dark matter haloes, it is also expected that the kinematic evolution of galaxies is influenced by the halo in which they reside.

However, galaxies and their haloes also feel the influence of the environment. Haloes falling towards and eventually orbiting within galaxy clusters are disrupted by a series of processes predominant in the cluster environment, for example, including the following: ram-pressure stripping (Gunn \& Gott
1972; Abadi et al. 1999; Bahé \& McCarthy 2015; Arthur et al. 2019; Mostoghiu et al. 2021) that removes the gas in haloes and quenches the star formation of galaxies; galaxy harassment (Moore et al. 1996, 1998; Smith et al. 2010, 2015), mergers (Dressler 1980; Hashimoto \& Oemler 2000; Behroozi et al. 2014), tidal torques (Fujita 1998; Balogh et al. 2000; Park et al. 2007), and interactions in general (Knebe et al. 2006; Recchi 2014) that can disrupt the haloes' components; and dynamical friction (Valtonen et al. 1990; Jiang \& Binney 2000; Fujii et al. 2006; van den Bosch 2017; Miller et al. 2020) which slows down infalling haloes and, over time, causes them to fall to the centre of the cluster. While the stars residing deep inside the potential well of the halo are shielded from tidal effects, they might nevertheless feel and react to the change of their own halo caused by the aforementioned processes. Or put differently, if the kinematic evolution of galaxies is indeed tied to their halo, the cluster environment affecting the halo could eventually also disrupt the kinematics of galaxies at their centre. This question now lies at the heart of the present study.

Previous numerical studies have shown how tidal interactions can disrupt infalling haloes in different environments. 
The seminal work of Hayashi et al. (2003) studied how tides influence substructures in dark matter-only simulations. Their analysis showed that, although tides preferentially strip the outer regions of haloes, they also decrease the halo's central density after each pericentric passage. Subsequent studies improved the tidal disruption estimation by including a stellar component to their analysis (e.g. Bullock \& Johnston 2005; Peñarrubia et al. 2008). The stellar component was found to be exceptionally resilient to tides, preserving its density profile shape even after losing a considerable number of stars. Nevertheless, the stellar dynamics of such calculations were modelled by analytic profiles, which simplify processes such as the mass loss from dynamical friction, the halo phase-space evolution after merger interactions, or the stellar mass fractions determined by star formation. More recent studies overcame these limitation by introducing a stellar component modelled by full-physics hydrodynamics (e.g. Smith et al. 2016; Łokas 2020; Errani \& Peñarrubia 2020; Mazzarini et al. 2020). However, these studies primarily focus on mass-loss processes, hence the question of how environmental effects influence the kinematic properties of the stellar component of infalling haloes has yet to be addressed.

We approach these issues by analysing simulations from THE THREE HUNDRED project ${ }^{1}$, that is a sample of over 300 galaxy clusters simulated with full-physics hydrodynamics (Cui et al. 2018). These simulated clusters have been used for different studies, for example, environmental effects (Wang et al. 2018), cluster profiles (Mostoghiu et al. 2019; Li et al. 2020; Baxter et al. 2021), backsplash galaxies (Arthur et al. 2019; Haggar et al. 2020; Knebe et al. 2020), the cluster dynamical state (Capalbo et al. 2021; De Luca et al. 2021), filament structures (Kuchner et al. 2020, 2021; Rost et al. 2021), and gravitational lensing (Vega-Ferrero et al. 2021). In this work we extend the previous stellar angular momentum analysis to this set of massive simulated galaxy clusters to study the influence of the cluster environment on the stellar kinematics of infalling galaxies.

This paper is organised as follows. In Sect. 2 we present the data used for the analysis. Section 2.1 briefly describes the simulations. In Sects. 2.2-2.4, we define how we selected the sample of galaxies used for the analysis and we present the classification of our objects. Section 3 describes our results: In Sect. 3.2 we focus on the kinematic evolution of the sample, and in Sect. 3.3 we study their angular momentum-stellar mass relation. Finally, we conclude the study in Sect. 4.

\section{The data}

\section{1. 'The Three Hundred' central galaxy clusters}

The simulations. The simulated clusters in THE THREE HUNDRED dataset were created by extracting 324 spherical regions of $15 h^{-1} \mathrm{Mpc}$ radius centred on each of the most massive haloes identified at $z=0$ within the dark-matter-only MDPL2 simulation (Klypin et al. 2016) ${ }^{2}$. MDPL2 was simulated with a Planck 2015 cosmology (Planck Collaboration XIII 2016), with $\Omega_{\mathrm{M}}=0.307, \Omega_{\mathrm{b}}=0.048, \Omega_{\Lambda}=0.693, h=0.678, \sigma_{8}=0.823$, and $n_{\mathrm{s}}=0.96$, and it consists of a box of $1 h^{-1} \mathrm{Gpc}$ sidelength which contains $3840^{3}$ dark matter particles each with a mass of $1.5 \times 10^{9} h^{-1} M_{\odot}$. In order to model the galaxy clusters with all the relevant baryonic physics, those $15 h^{-1} \mathrm{Mpc}$

\footnotetext{
1 https://the300-project.org

2 The MultiDark simulations, including the MDPL2 used here, are publicly available at https://www. cosmosim.org
}

regions were traced back to the initial conditions and were populated with gas particles there by leading to a mass resolution of $m_{\mathrm{DM}}=1.27 \times 10^{9} h^{-1} M_{\odot}$ and $m_{\mathrm{gas}}=2.36 \times 10^{8} \mathrm{~h}^{-1} M_{\odot}$, respectively. Outside the re-simulated region, to reduce the computational cost of the original MDPL2 simulation, dark matter particles were degraded with lower mass resolution particles to maintain the same large-scale tidal field. Using a Plummer equivalent softening of $6.5 \mathrm{~h}^{-1} \mathrm{kpc}$ for both the dark matter and baryonic component, the new initial conditions were then moved forward in time using GADGET-X (Beck et al. 2016). GADGET-X is a modified version of GADGET3 with a modern smooth particle hydrodynamics (SPH) scheme which improves the treatment of gas particles (Beck et al. 2016; Sembolini et al. 2016a). The results of simulations of galaxy clusters based on GADGET-X have been presented in several previous papers (e.g. Rasia et al. 2015; Planelles et al. 2017) and in the NIFTY cluster comparison project (Sembolini et al. 2016b; Elahi et al. 2016; Cui et al. 2016; Arthur et al. 2017). A total of 129 snapshots have been saved from $z=16.98$ to $z=0$.

The halo finding. The halo analysis was done using the $\mathrm{AHF}^{3}$ halo finder (Gill et al. 2004; Knollmann \& Knebe 2009). AHF locates local overdensities in an adaptively smoothed density field as potential halo centres and automatically identifies haloes and a substructure (subhaloes, subsubhaloes, etc.). The radius of a halo $R_{200}$ and the corresponding enclosed mass $M_{200}$ were calculated as the radius $r$ at which the cumulative density $\rho(<r)=M(<r) /\left(4 \pi r^{3} / 3\right)$ dropped below $200 \rho_{\text {crit }}(z)$, where $\rho_{\text {crit }}(z)$ is the critical density of the Universe at a given redshift $z$.

The merger trees. The progenitors of the haloes were tracked across the snapshots with MergerTree, a tool that comes with AHF. Each halo identified at redshift $z=0$ was tracked backwards in time, identifying, as the main progenitor at some previous redshift, the halo that maximises the merit function $\mathcal{M}=N_{A \cap B}^{2} /\left(N_{A} N_{B}\right)$, where $N_{A}$ and $N_{B}$ are the number of particles in haloes $H_{A}$ and $H_{B}$, respectively, and $N_{A \cap B}$ is the number of particles that are in both $H_{A}$ and $H_{B}$. The code further has the ability to skip snapshots, that is to say progenitors of haloes that are not found in the previous snapshot are still searched for in earlier snapshots, recovering an otherwise truncated branch of the merger tree (see Wang et al. 2016). However, to reduce errors during the tracking of the stellar kinematic evolution of our objects, we consider an object to be 'lost' if it cannot be found for five consecutive snapshots.

In summary, in our study there are 324 numerically modelled central galaxy clusters and all the haloes orbiting in and about them out to a distance of $15 h^{-1} \mathrm{Mpc}$ are available for our analysis. The details of this full data set are presented in Cui et al. (2018). However, for the purposes of this work, we applied a few selection criteria to both the central clusters and the field haloes as well as subhaloes in the regions, and this is described below.

\subsection{Cluster selection}

As we aim to trace back all the objects in and about the central galaxy cluster, our analysis requires that we can always define a main progenitor for each of the 324 central haloes. As shown in Behroozi et al. (2015), major mergers during the formation of those central objects pose a challenge to this. Following the discussion in Haggar et al. (2020), we therefore identified and removed central cluster haloes whose main

\footnotetext{
http://popia.ft.uam. es/AHF
} 
progenitor's position changes by more than half their radius $R_{200}(z)$ between two consecutive snapshots $[z, z+\Delta z]$. We further required the main branch to at least extend to a redshift of $z=2$. This reduces the number of regions entering the analysis from 324 down to 236 . There are approximately 8 times more cluster regions used here than in other state-of-the-art cluster simulation studies (e.g. Bahé et al. 2017; Barnes et al. 2017).

\subsection{Halo selection}

Each of our 236 selected central galaxy clusters is surrounded by a multitude of haloes out to the $15 h^{-1} \mathrm{Mpc}$ edge of the region that was modelled including all the relevant baryonic physics. But as we are interested in studying the angular momentum of the stellar component of these haloes, we limited our analysis to haloes with at least $M_{\text {star }}>5 \times 10^{10} h^{-1} M_{\odot}$. This corresponds to at least 1000 star particles. Further, only those haloes that lie within $3 R_{200}$ of the central galaxy cluster at a redshift of $z=0$ were traced backwards in time. For these haloes we then define their infall redshift $z_{\text {inf }}$ as the redshift at which the halo crosses central galaxy cluster's $2 R_{200}(z)$ for the first time: Recent numerical studies show that around $\sim 1.5 R_{200}-2 R_{200}$, haloes experience a sharp cut-off in their gas content which could indicate the presence of an accretion shock (Power et al. 2020; Arthur et al. 2019; Baxter et al. 2021) and hence we decided to use $2 R_{200}$ (instead of $R_{200}$ ) as our reference crossing radius. Haloes that cannot be assigned an infall redshift have been removed from the analysis.

\subsection{Stellar component}

To select the stellar component of our objects that one might identify with their central galaxy, we opted for a spherical region enclosing $15 \%$ of the haloes' physical radius (e.g. Bailin \& Steinmetz 2005). However, as tidal interactions with the central galaxy cluster impact the size of subhaloes (Muldrew et al. 2011; Onions et al. 2012), we used the radius as found right before crossing $2 R_{200}$ of the central galaxy cluster. This aperture is now interpreted as the 'size of the galaxy' and was kept fixed in physical coordinates across the snapshots. We performed a series of tests using different criteria (e.g. an aperture not depending on the halo's physical radius, but rather fixing it to $30 \mathrm{kpc}$ ) that showed that even though haloes suffer an overall mass loss while orbiting within their host halo, the central stellar mass in the aperture is mostly unaffected. This agrees with other simulations, which show that stellar stripping is rare, and it happens only after the dark matter has suffered significant stripping (>80\%, e.g. Smith et al. 2016; Bahé et al. 2019). It is important to point out again that even though the stellar mass of our galaxy does not change (something we also quantify below), we cannot rule out any back-reaction of the stars to the change in potential caused by tidal stripping of the halo. Finally, to take the spatial resolution of our simulations into account, we restricted our objects to have a 'galaxy size' of at least $2 \epsilon=13 h^{-1} \mathrm{kpc} \sim 20 \mathrm{kpc}$. We remark that this additional condition removes only the smallest galaxies in the sample, which is less than $5 \%$ of the objects selected so far. Moreover, practically all of the star particles reside within our aperture and hence we did not considerably reduce the number of star particles by cutting out the 'galaxy' as defined here. The remaining 6509 objects, with halo masses from $9.2 \times 10^{10} h^{-1} M_{\odot}$ to $5.3 \times 10^{14} h^{-1} M_{\odot}$, constitute the analysis sample, built by combining all the objects that satisfy the aforementioned criteria from the 236 cluster regions considered here.
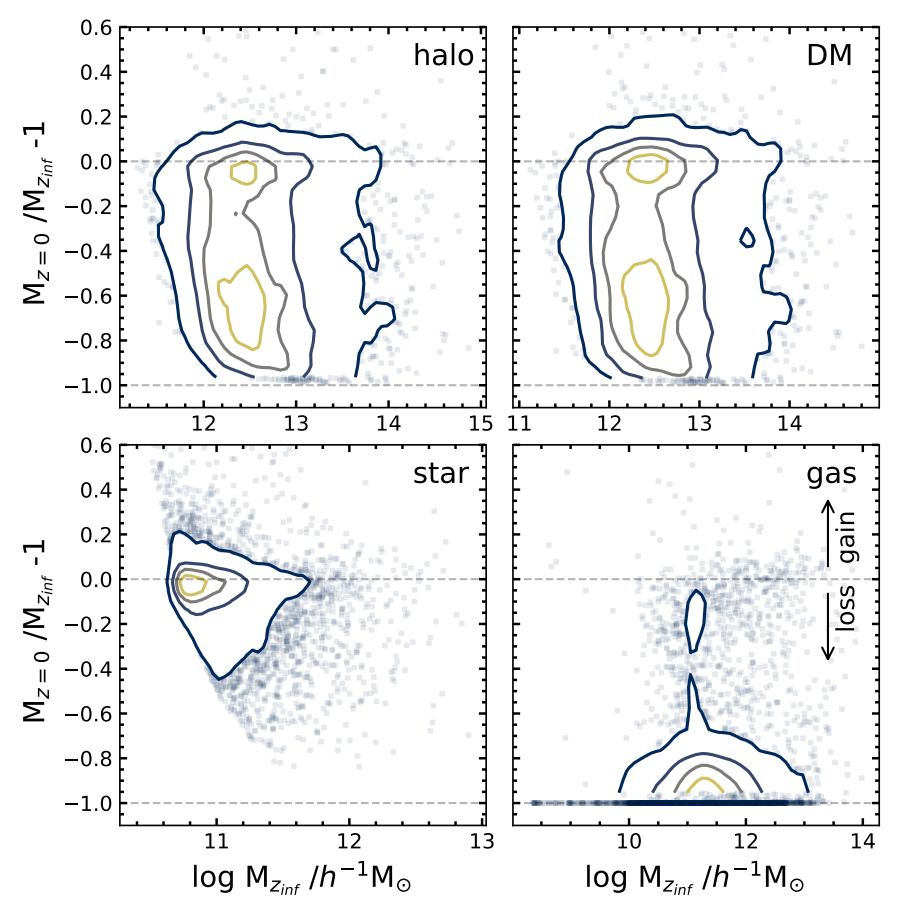

Fig. 1. Evolution of the different mass components of the haloes in the sample from their infall redshift $z_{\text {inf }}$ to their $z=0$ values. Contour levels show the $75,50,25$, and $5 \%$ of the maximum counts of the distributions. The horizontal lines at 0 and -1 correspond to no change and maximum mass loss, respectively. The positions in the plane of objects beyond the $5 \%$ contour are shown as square markers.

\section{Results}

In what follows, we compare the properties of our selected haloes orbiting in and about the central galaxy clusters at infall redshift $z_{\text {inf }}$ (i.e. when crossing $2 R_{200}$ ) and present-day time $z=0$.

\subsection{Mass evolution}

To understand the processes that shape the haloes after their first infall, we show in Fig. 1 the fractional change in mass since their infall redshift and as a function of infall mass. Contour levels show $75,50,25$, and $5 \%$ of the maximum counts of the distributions. The horizontal lines at 0 and -1 correspond to no change and maximum mass loss, respectively. Objects beyond the 5\% contour are shown as square markers. As expected, haloes crossing the denser regions of the central galaxy cluster lose a considerable amount of mass (e.g. Klimentowski et al. 2010). Haloes that only entered the central halo once and did not leave, in general, conserve more of their initial mass than the ones that experienced multiple infalls into the denser regions of the galaxy cluster. In terms of the stellar mass in the haloes, the loss is significantly lower due to the fact that most of the stellar component resides in the central region of the halo mainly shielded from tidal forces. We nevertheless observe an increase in stellar mass loss at the 5\% level as we move to the massive end of the stellar mass distribution. Such haloes present substructure residing in the halo component (i.e. outside the central aperture) and thus they are prone to suffer from mass loss from processes that strip the halo at its outskirts. The gas component, on the other hand, is mostly gone regardless of their mass, as gas is affected by an entirely different set of processes as they fall into the central halo, that is to say ram-pressure stripping (Arthur et al. 2019; 
Mostoghiu et al. 2021). In studying the radial distribution of the gas inside infalling haloes (not shown here), we find that haloes that never reached the denser regions of the cluster halo are still considerably stripped of their gas component, which is in agreement with Power et al. (2020), Arthur et al. (2019), and Mostoghiu et al. (2021).

In summary, Fig. 1 clearly shows that the stellar component of our haloes is the least affected by the environment of the central galaxy cluster. The question nevertheless remains if we will find changes in the kinematical properties. But the haloes of our 'galaxies' (as defined by the star particles in the aperture, cf. Sect. 2.4) certainly undergo some changes as manifested by the mass loss. It is therefore not yet clear why even though the galactic stellar mass more or less remains constant that there is no reaction of its internal dynamics to the varying influence of the central galaxy cluster.

\subsection{Fraction of co-rotational energy}

We study the influence of the central galaxy cluster on the internal dynamics of our galaxy sample after the sample's first infall by following the evolution of its specific stellar angular momentum $j_{*}$.

The galaxies in our sample can be classified by the fraction of stellar kinetic energy that is invested in co-rotation $\kappa_{\text {corot }}$, as presented in Sales et al. (2010), and Correa et al. (2017):

$\kappa_{\mathrm{corot}}=\frac{E_{\mathrm{corot}}}{E_{\mathrm{kin}}}, E_{\mathrm{corot}}=\sum_{j_{z, i}>0} \frac{1}{2} m_{i}\left(\frac{j_{z, i}}{r_{2 d, i}}\right)^{2}$,

where $E_{\text {kin }}$ is the kinetic energy of the star particles in the aperture; $E_{\text {corot }}$ is the rotational energy of the corotating star particles contributing to the rotation of the galaxy; $m_{i}$ is the mass of the star particle;

$j_{z, i}=\boldsymbol{j}_{i} \cdot \hat{\boldsymbol{J}}_{\mathrm{tot}}$,

$\boldsymbol{j}_{i}=\left(\boldsymbol{r}_{i}-\boldsymbol{r}_{\text {halo }}\right) \times\left(\boldsymbol{v}_{i}-\boldsymbol{v}_{\text {halo }}\right)$, and

$\hat{\boldsymbol{J}}_{\mathrm{tot}}=\frac{1}{J_{\mathrm{tot}}} \frac{\sum m_{i} \boldsymbol{j}_{i}}{\sum m_{i}}$

are the specific angular momentum along the direction of the total angular momentum, the specific angular momentum of a star particle in the rest frame of its halo, and the direction of the total specific angular momentum of the galaxy, respectively; and $r_{2 d, i}=\left(\left|\boldsymbol{r}_{i}-\boldsymbol{r}_{\text {halo }}\right|^{2}-\left(\left(\boldsymbol{r}_{i}-\boldsymbol{r}_{\text {halo }}\right) \cdot \hat{\boldsymbol{J}}_{\text {tot }}\right)^{2}\right)^{1 / 2}$ is the cylindrical radius of star particles. As we exclusively use the definition based on co-rotating star particles, we cease to write the subscript of the $\kappa$ parameter from now on.

\subsubsection{Changes in $\kappa$ since the infall}

We compared the fraction of ordered rotation at two different times in the evolution of the galaxies to find if the $z=0$ values are the result of the influence of the central galaxy cluster during their infall. In Fig. 2 we show the $\kappa$ values of the sample at $z=0$ compared to their values at infall $z_{\text {inf }}$, before experiencing the central cluster's influence. The black dashed diagonal line shows the $1: 1$ relation. The median values in bins of $\kappa$ at $z=0$ are represented by the solid cyan line. Bins are coloured by their corresponding number of counts and contours show where 75 , 50,25 , and $5 \%$ of the maximum counts lie.

We find that for most of the haloes in the sample, the fraction of stellar ordered rotation is unaffected by their infall (Pearson

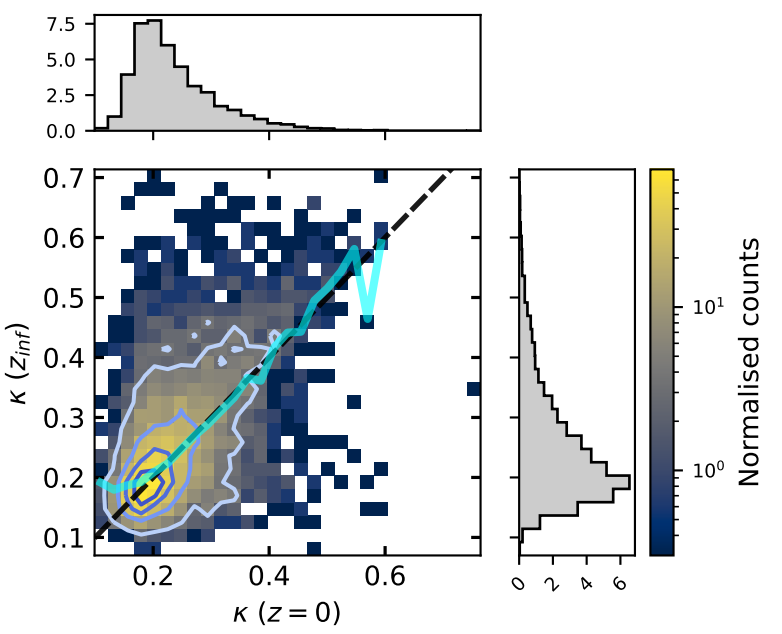

Fig. 2. Fraction of ordered rotation $\kappa$ at $z=0$ and at the infall redshift $z_{\text {inf }}$. The diagonal dashed line shows the 1:1 relation. Median values in bins of $\kappa(z=0)$ for the sample are represented by the solid cyan line. Bins are coloured by their corresponding normalised counts. The contour lines show where $75,50,25$, and $5 \%$ of the maximum counts lie.
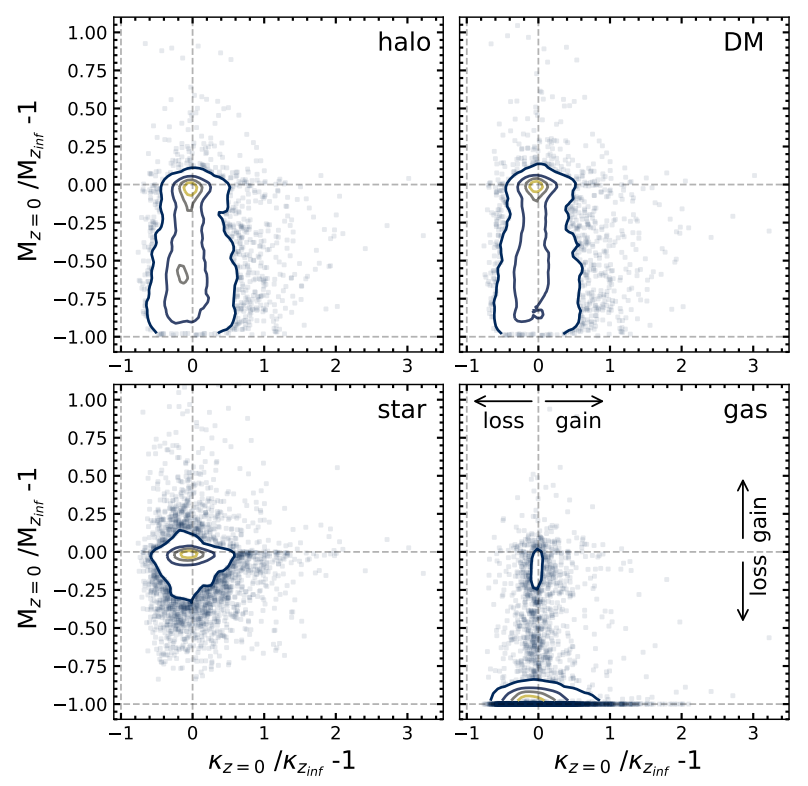

Fig. 3. Evolution of the different mass components of the haloes in the sample and their fraction of ordered rotation $\kappa$ from their infall redshift $z_{\text {inf }}$ to their $z=0$ values. Contour levels show the 75, 50, 25, and 5\% of the maximum counts of the distributions. The horizontal lines at 0 and -1 correspond to no change and maximum mass loss, respectively. The position in the plane of objects beyond the $5 \%$ contour are shown as square markers.

coefficient of 0.56), with a median value at $z=0$ and at $z_{\text {inf }}$ of $\kappa \sim 0.22$. However, beyond the $5 \%$ contour, we identify galaxies which experienced a considerable change in $\kappa$ since their infall, for example $\Delta \kappa \sim \pm 0.2-0.4$.

In Sect. 3.1 we found that the haloes in the sample are stripped of their material during their fall into the denser regions of the central galaxy cluster. Nevertheless, mass loss does not appear to considerably affect the rotational properties of their galaxies, as seen from their $\kappa$ parameter. We quantify this change in Fig. 3, in which we show the same evolution of the mass component of the haloes in the sample presented in Fig. 1, but this 
time as a function of the evolution in their fraction of ordered rotation.

For the total halo and DM mass components of the haloes in the sample, we identified objects that lost up to $90 \%$ of their mass at infall, yet we did not find a correlation between the amount of mass loss and their fraction of ordered rotation evolution trend. At the $5 \%$ contour, regardless of the amount of stripped mass, their fraction of stellar ordered rotation changed within $\sim \pm 60 \%$ since their infall. As we have discussed in the previous section, the stellar component is less affected by mass-loss processes. Indeed, we see that at the 5\% contour, the objects experienced a change in stellar mass within $\sim \pm 30 \%$ and that their change in $\kappa$ tends towards zero as their infall mass is disrupted. This is likely due to the fact that such stellar mass gain (or loss) tends to happen in the outer region of haloes, outside the apertures used to define the central galaxy and calculate $\kappa$. As for the gas component, even when haloes have been mostly stripped of their gas by the time they reach $z=0$, the stellar fraction of ordered rotation does not correlate with such mass loss. Our galaxies can fall into galaxy cluster regions, losing most (if not all) of their gas during their infall, and they still retain the stellar kinematic properties they had prior to their infall. Similar results have been reported in Cortese et al. (2019): Satellite galaxies go through significant changes in their specific star formation rate, but they are not necessarily accompanied by changes in their stellar spin parameter.

Overall, we find no correlation between the amount of stripped mass and the change in the $\kappa$ parameter. Thus, we conclude that the fraction of ordered rotation in our simulations is hardly affected by the (potentially violent) stripping processes that disrupt the dark matter halo.

\subsubsection{Temporal evolution of high and low $\kappa$ galaxies}

In Fig. 2 we identified objects beyond the 5\% contour with a considerable change in their fraction of stellar co-rotational energy since their infall. To study which processes are responsible for such changes, from the $\kappa$ distribution at $z=0$, we selected the galaxies within $5 \%$ of the highest and lowest fractions of ordered rotation, that is the $\kappa>95$ th percentile, which is the high $\kappa$ sub-sample, and the $\kappa<5$ th percentile, which is the low $\kappa$ subsample of the distribution. This corresponds to a high $\kappa$ threshold of 0.38 and a low $\kappa$ threshold of 0.15 . We note that our choice for the threshold values is purely motivated by kinematics: We simply aim to understand the origin of the substantial changes in the $\kappa$ distribution of a (relatively) small fraction of the total sample. Adopting other threshold values, which might also take into account other non-kinematic properties such as star formation or colours (for example $\kappa=0.4$, from Correa et al. 2017) for the stellar kinematic classification, only $4 \%$ of our sample would be classified (in terms of their kinematics) as fast-rotating galaxies.

Recent numerical results show that galaxies which continue to accrete gas and form stars are very efficient at spinning up (Lagos et al. 2017). However, this is unlikely to be the cause here as gas accretion is expected to be hampered in clusters. Lagos et al. (2018a) found that galaxies can be spun up or down by mergers depending on their orbital orientation and gas content (see Schulze et al. 2018; Lagos et al. 2018b). To investigate the origin of the change in the fraction of co-rotation after infall found in Fig. 2, in Fig. 4 we study the $\kappa$ evolution of the low and high $\kappa$ sub-samples found at $z=0$ as a function of the time since their pericentre passage, defined as the closest approach of an
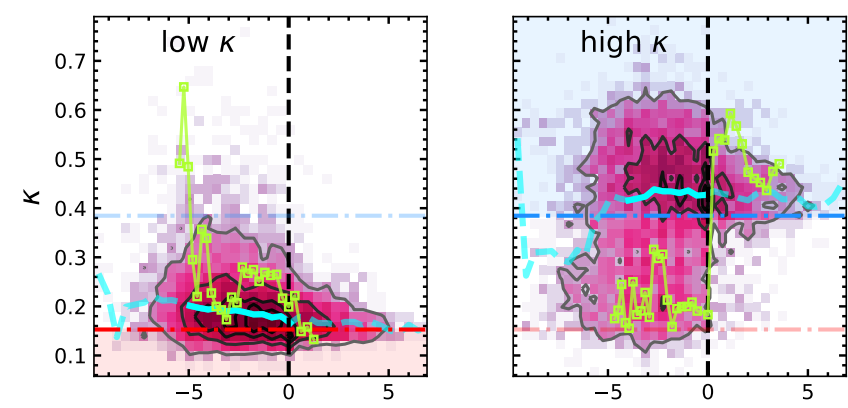

time since pericentre [Gyr] time since pericentre [Gyr]

Fig. 4. Evolution of the fraction of co-rotational energy as a function of the time since the pericentre passage for low (left) and high (right) $K$ subsample as classified by the 5th and 95th percentiles of the $z=0 \kappa$ distribution in Fig. 2. Contours show 75, 50, 25, and 5\% of the maximum counts of the $2 \mathrm{D}$ distribution. The cyan solid line shows the median value at each time bin. For bins in which less than $50 \%$ of the sample entered the median calculation, we instead use a dashed line. The red and blue horizontal dash-dotted lines show the $\kappa$ threshold values for the low and high $\kappa$ sub-samples, respectively. The pericentre is marked with a vertical dashed line. In each panel we additionally show the evolution of a galaxy from the corresponding sub-sample using green square markers. The shaded red and blue regions show the allowed $\kappa$ values corresponding to each sub-sample.

infalling halo to the central galaxy cluster ${ }^{4}$. Contours show 75 , 50,25 , and $5 \%$ of the maximum counts in the distribution. We show the median value at each time bin with a cyan solid line, and when the number of haloes in a bin is less than $50 \%$ of the maximum count we use a dashed line instead. The low and high $\kappa$ threshold values are represented by the red and blue horizontal dash-dotted lines, respectively, and the pericentre time is marked with a vertical dashed line. In each panel we additionally show the $\kappa$ evolution of a galaxy from the respective sub-sample with green square markers, and we marked the allowed $\kappa$ values of each sub-sample with corresponding shaded regions.

As already anticipated by the $\kappa$ evolution in Fig. 2, most of the galaxies in each sub-sample retain their fraction of co-rotational energy $\kappa$ after their infall. However, over the course of $5 \mathrm{Gyr}$ after the pericentre passage, we observe a decrease of $\sim 0.2$ in $\kappa$ in both sub-samples $\left(\sim 0.04 \mathrm{Gyr}^{-1}\right)$ at the $5 \%$ contour. Moreover, the $\kappa$ evolution of the low $\kappa$ sample galaxy shows that it had high stellar co-rotational energy at some point, but lost it after $\sim 6$ Gyr. Such a slow decrease can be attributed to the twobody heating of the stellar component: As galaxies infall into the central galaxy cluster, the background (more massive) dark matter particles residing inside the cluster that fly by these galaxies tend to increase the stellar mean interparticle distance due to the softening scale used for the stellar particles, heating up the stellar component and effectively puffing up the stellar distribution while spinning them down. As these galaxies fall further into the cluster, the density of dark matter particles increases and consequently the amount of fly-bys is boosted. Along with this slow decrease, we also identify processes which instead can spin up galaxies on a faster scale. But these changes, as observed for the high $\kappa$ sample galaxy, occur close to pericentre passage and are investigated in more detail below.

4 Our definition of 'pericentre' does not necessarily imply that infalling objects are within $R_{200}$ of the central galaxy cluster. 


\subsubsection{Spinning-up galaxies with low $\kappa$}

As two-body heating slows down the stellar kinematics of every infalling galaxy in equal measure, that is to say $\kappa$ decreases at the same rate for both sub-samples, we focus on processes which are able to spin up galaxies during their infall. To isolate the galaxies that cross the threshold $\kappa$ value to become high $\kappa$ galaxies according to our classification from the ones that conserve their classification up to $z=0$, that is to say the ones that remained within the high $\kappa$ sub-sample after their infall into the cluster environment, we selected galaxies from the high $\kappa$ sub-sample which experienced a rapid $\kappa$ increase (i.e. $\Delta \kappa>0.2$ ) within $1 \mathrm{Gyr}$ since their pericentre passage. These galaxies form only $12 \%$ of the high $\kappa$ galaxies at $z=0$ (39 objects, $0.6 \%$ of the total number of objects in the sample). Within this new sub-sample, we identified galaxies for which a sudden change in their star particle count was accompanied by a sudden spin-up of their specific angular momentum (and consequently an increase in their $\kappa$ parameter) near their pericentre, and galaxies for which the number of stars close to the pericentre does not seem to play a crucial role in their $\kappa$ evolution.

Following the star particle distribution of these galaxies, we find that the fast increase in $\kappa$ and in the number of stars is a consequence of two processes: mergers with other haloes and flybys of stellar remnants within the central aperture used to define the galaxies residing in each halo. In agreement with Lagos et al. (2018a), we find that the co-rotating (counter-rotating) infalling stars from mergers are able to spin up (spin down) our galaxies. On the other hand, acting on shorter timescales and without a significant stellar gain, we find that stellar remnants are able to temporarily disrupt the spin of our objects. These haloes are the residual cores of infalling haloes, where the original dark matter component of the halo has become lost and subsumed by the main halo. Considering that the stellar component of the remnant haloes is smaller than the central galaxy extension of the haloes in the sample $(\sim 1 / 3$ of the aperture size), and that the amount of remnant stellar cores increases close to the centre of the galaxy cluster, these objects contribute transiently to the co-rotational energy in the aperture and, as such, $\kappa$ increases near the pericentre of our objects.

\subsubsection{Summary}

We conclude that for most of the galaxies in our sample entering massive galaxy clusters, their kinematic properties (as captured by the fraction of ordered rotation $\kappa$ ) do not change in a significant manner, even though we have seen in Sect. 3.1 that their haloes undergo substantial changes. In general, low $\kappa$ galaxies at $z=0$ had low fractions of ordered rotation before entering the cluster halo, and high $\kappa$ galaxies at $z=0$ had such high fractions prior to entering the cluster environment. The two-body heating of the stellar component of infalling haloes induced by the more massive dark matter particles in the cluster environment affects both samples equally, slowly reducing their stellar specific angular momentum as they orbit the cluster region. As such, we find that galaxies that had high fractions of stellar co-rotational energy $(\kappa \gtrsim 0.4)$ at their infall time ended up as low $\kappa$ galaxies by the time they reached $z=0$, that is with a $\kappa<5$ th percentile of the $\kappa$ sample distribution at $z=0$. On the other hand, from the high $\kappa$ sample at $z=0$, that is the galaxies with $\kappa>95$ th percentile, we identify $\sim 12 \%$ of them which previously had considerably lower fractions. We find that these two processes acting on different timescales, that is to say mergers with other infalling haloes and stellar remnants transiting the aperture

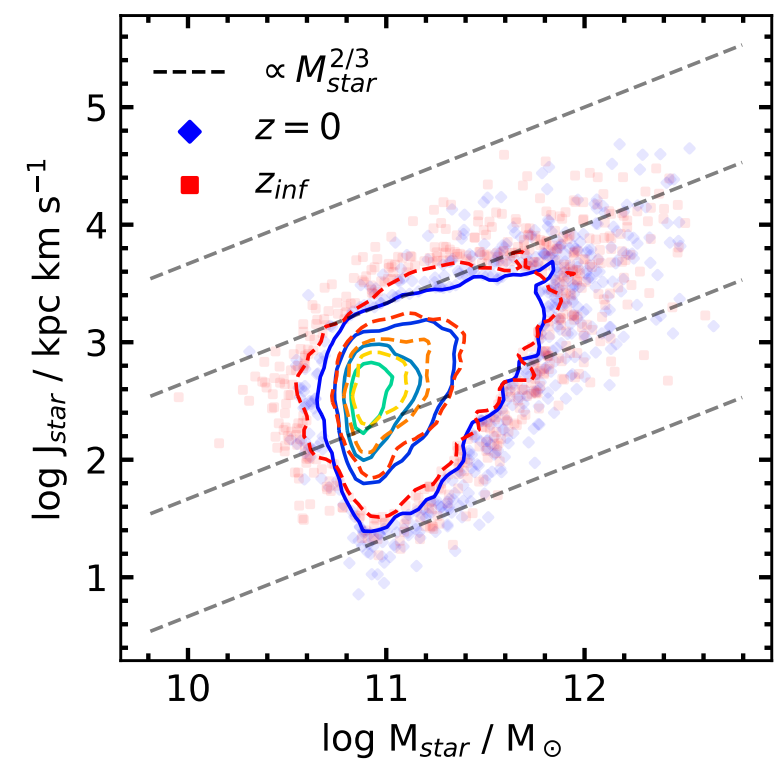

Fig. 5. Specific angular momentum and stellar mass relation of the sample of galaxies at $z=0$ and at their infall redshift $z_{\text {inf }}$. The diagonal dashed lines show the $M_{\text {star }}^{2 / 3}$ relation for zero-points -3 (top line) to -6 (bottom line). The contours show 75, 50,25, and 5\% of the sample. The positions in the plane of objects beyond the $5 \%$ contour are shown as filled markers.

used for defining the galaxies of the haloes in the sample, are able to spin up the galaxies.

\subsection{Stellar angular momentum and stellar mass relation}

In the previous section we identified processes which are able to change specific angular momentum of infalling galaxies. Nevertheless, most of the objects remain unaffected by such interactions. In this section we study the influence of the central galaxy cluster on the internal dynamics of our galaxies by following their evolution in the specific angular momentum $j_{\text {star }}-$ stellar mass $M_{\text {star }}$ plane, an extensively studied fundamental kinematic property of galaxies which can be described via a power-law of the form $j_{\text {star }} \propto M_{\text {star }}^{\alpha}$, where $\alpha \approx 0.7$ (e.g. Fall 1983; Romanowsky \& Fall 2012; Obreschkow \& Glazebrook 2014; Teklu et al. 2015; Fall \& Romanowsky 2018).

As some of our galaxies experienced great changes in their fraction of ordered rotation during their evolution since their infall, we aim to find if these changes are also visible in the specific stellar angular momentum-stellar mass plane. We show their position in the $j-M$ plane after their infall redshift in Fig. 5. The contours represent $75,50,25$, and $5 \%$ of the sample. The position in the plane of galaxies beyond the $5 \%$ contour are shown as filled markers. The dashed lines indicate the $j_{\text {star }}=\lambda M_{\text {star }}^{2 / 3}$ relation for different zero-points $(\log \lambda=-3$ to -6), as presented in Teklu et al. (2015). We note that, overall, the sample in this analysis does not include galaxies with stellar specific angular momenta typical of disk galaxies in the local Universe.

Once again, we find that the distribution is mostly unchanged after entering the cluster halo. However, galaxies positioned furthest from the average position in the $j-M$ plane at their infall redshift move towards lower specific angular momentum and stellar mass values after infalling, as seen in the 5\% contours and points below that level. These results show that the internal dynamics of our infalling galaxies into massive galaxy clusters, 
as quantified by either the fraction of ordered rotation $\kappa$ or the evolution of their location in the specific angular momentumstellar mass plane, are mostly unaffected by the processes in such environments even after their halos have been considerably stripped of their mass and, consequently, have disrupted the halo potential in which these galaxies reside.

\section{Conclusions}

We analysed the central stellar component of haloes from THE THREE HUNDRED project, a suite of 324 galaxy cluster regions simulated with full-physics hydrodynamics, to study of the influence of galaxy clusters on the internal dynamics of infalling galaxies. We selected objects with $M_{\text {star }}>5 \times 10^{10} h^{-1} M_{\odot}$ within $3 R_{200}$ from the galaxy cluster halo at $z=0$. After applying different selection criteria that ensure the correct tracking of the stellar component of each halo, we obtained a sample of 6509 galaxies from 236 cluster regions, that is to say approximately 8 times more cluster regions than used in other state-of-the-art cluster simulation studies (e.g. Bahé et al. 2017; Barnes et al. 2017). Here we summarise our main results:

- Using the fraction of stellar co-rotational energy $\kappa$ to track the internal dynamics of the infalling galaxies in our sample, we find that the sample's stellar dynamics remains mostly unchanged for most of the galaxies after they enter the galaxy cluster environment, despite the (potentially violent) stripping processes that disrupt their haloes.

- We studied the time evolution of the $\kappa$ parameter. Even though most galaxies do not experience great $\kappa$ changes during their evolution, we identify an overall slow decrease in the fraction of ordered rotation close to their pericentre passage $\left(\sim 0.04 \mathrm{Gyr}^{-1}\right)$, which we attribute to numerical effects. Along with this effect, we found that $\sim 12 \%$ of the galaxies with high $\kappa$ at $z=0$ (i.e. $\kappa>95$ th percentile of the $z=0$ distribution), an already small population in our sample, had considerably lower fractions of co-rotational energy in the past and experienced a fast boost in their $\kappa$ parameter within the past few gigayears $\left(\sim 0.2 \mathrm{Gyr}^{-1}\right)$ due to two different processes: stellar mergers of infalling haloes (in agreement with Lagos et al. 2018a,b) and transient encounters with the stellar remnants of haloes that have lost their dark matter component during their own passage through the cluster, entering within the aperture we used for defining the properties of our galaxies.

- We found that similar to the $\kappa$ evolution of our galaxies, the specific angular momentum-stellar mass relation for the galaxies in the sample showed no substantial change in their location on the $j-M$ plane from their infall redshift until $z=0$.

Previous numerical studies (e.g. Smith et al. 2016; Łokas 2020) have shown that infalling and orbiting haloes within galaxy clusters are disrupted by a series of processes which dominate in such environments, such as ram-pressure stripping (e.g. Bahé \& McCarthy 2015; Arthur et al. 2019; Mostoghiu et al. 2021), mergers (e.g. Behroozi et al. 2014; Lagos et al. 2018a), or tidal torques (e.g. Park et al. 2007). Nevertheless, these numerical studies primarily analyse mass-loss processes in infalling haloes. Considering that cluster environments disrupt the different mass components of infalling haloes, such environments could also potentially disrupt their stellar kinematics.

In this work we investigate such a question by studying the internal central stellar dynamics of infalling objects towards the numerically simulated massive galaxy clusters in our sample. We found that, roughly, their $z=0$ classification holds even from before the time they entered the cluster environment. Moreover, we find that in terms of their position in the fundamental specific stellar angular momentum-stellar mass plane (e.g. Obreschkow \& Glazebrook 2014; Fall \& Romanowsky 2018), they remained mostly unaffected by such processes. For a small number of galaxies, we identified processes which are able to considerably boost the spin of infalling galaxies during their infall via mergers with other objects and via transient encounters with stellar remnants within the cluster halo. These two processes are able to offset the numerical slow-down induced by numerical effects, such that at $z=0$ they end up within the top $5 \%$ galaxies with the highest amount of ordered rotation within the simulation.

Due the mass resolution of our simulations, which favours the modelling of a large number of galaxy clusters and their environments, our sample contains a limited kinematic distribution of galaxies when compared with observations (e.g. Brough et al. 2017). Thus, generalising these environmental constrains to galaxies with higher stellar angular momentum is not possible, as mass-loss processes during the infall of such galaxies in cluster environments might prove to play a more crucial role in their stellar angular momentum evolution. Future simulations, where the stellar mass resolution is increased and a smaller softening scale is used to better resolve the stellar component, for similar cosmological volumes, would help us extend our analysis to a wider range of galaxies with higher fractions of ordered rotation and obtain environmental constraints with far greater statistical significance.

Acknowledgements. This work has been made possible by the 'The Three Hundred' Collaboration (https://www. the300-project.org). The project has received financial support from the European Union's Horizon 2020 Research and Innovation programme under the Marie Sklodowskaw-Curie grant agreement number 734374 , i.e. the LACEGAL project. The authors would like to thank the anonymous referee for their constructive feedback, which improved the quality of this paper. Additionally, they would like to thank The Red Española de Supercomputación for granting us computing time at the MareNostrum Supercomputer of the BSC-CNS where most of the cluster simulations have been performed. Part of the computations with GADGET-X have also been performed at the 'Leibniz-Rechenzentrum' with CPU time assigned to the Project 'pr83li'. $\mathrm{RM}, \mathrm{AK}$, and GY would like to thank MINECO/FEDER (Spain) for financial support under research grants AYA2015-63819-P and MICIU/FEDER for financial support under research grant PGC2018-094975-C21. RM further acknowledges support from the International Centre for Radio Astronomy (ICRAR) for funding this project. AK further acknowledges support from the Spanish Red Consolider MultiDark FPA2017- 90566-REDC and thanks Joy Division for unknown pleasures. CL has received funding from the ARC Centre of Excellence for All Sky Astrophysics in 3 Dimensions (ASTRO 3D), through project number CE170100013. WC acknowledges the supported by the European Research Council under grant number 670193. SB acknowledges financial support from PRIN-MIUR 2015W7KAWC, the INFN INDARK grant, and the EU H2020 Research and Innovation Programme under the ExaNeSt project (Grant Agreement No. 671553). KD acknowledges support through ORIGINS, founded by the Deutsche Forschungsgemeinschaft (DFG, German Research Foundation) under Germany's Excellence Strategy - EXC-2094 - 390783311. The authors contributed to this paper in the following ways: RM, AK, FRP and CP formed the core team. RM analysed the data, produced the plots and wrote the paper along with AK, FRP, CL, WC, SB, and KD. SB, KD, GM, and GY supplied the simulation data. All authors had the opportunity to provide comments on this work. This work was created by making use of the following software: Python, Matplotlib (Hunter 2007), Numpy (van der Walt et al. 2011), scipy (Virtanen et al. 2020), astropy (Astropy Collaboration 2013, 2018), and pynbody (Pontzen et al. 2013).

\section{References}

Abadi, M. G., Moore, B., \& Bower, R. G. 1999, MNRAS, 308, 947 Arthur, J., Pearce, F. R., Gray, M. E., et al. 2017, MNRAS, 464, 2027 Arthur, J., Pearce, F. R., Gray, M. E., et al. 2019, MNRAS, 484, 3968 Astropy Collaboration (Robitaille, T. P., et al.) 2013, A\&A, 558, A33 
Astropy Collaboration (Price-Whelan, A. M., et al.) 2018, AJ, 156, 123 Bahé, Y. M., \& McCarthy, I. G. 2015, MNRAS, 447, 969

Bahé, Y. M., Barnes, D. J., Dalla Vecchia, C., et al. 2017, MNRAS, 470, 4186

Bahé, Y. M., Schaye, J., Barnes, D. J., et al. 2019, MNRAS, 485, 2287

Bailin, J., \& Steinmetz, M. 2005, ApJ, 627, 647

Balogh, M. L., Navarro, J. F., \& Morris, S. L. 2000, ApJ, 540, 113

Barnes, D. J., Kay, S. T., Bahé, Y. M., et al. 2017, MNRAS, 471, 1088

Baxter, E. J., Adhikari, S., Vega-Ferrero, J., et al. 2021, ArXiv e-prints [arXiv:2101.04179]

Beck, A. M., Murante, G., Arth, A., et al. 2016, MNRAS, 455, 2110

Behroozi, P. S., Wechsler, R. H., Lu, Y., et al. 2014, ApJ, 787, 156

Behroozi, P., Knebe, A., Pearce, F. R., et al. 2015, MNRAS, 454, 3020

Brough, S., van de Sande, J., Owers, M. S., et al. 2017, ApJ, 844, 59

Bullock, J. S., \& Johnston, K. V. 2005, ApJ, 635, 931

Capalbo, V., De Petris, M., De Luca, F., et al. 2021, MNRAS, 503, 6155

Correa, C. A., Schaye, J., Clauwens, B., et al. 2017, MNRAS, 472, L45

Cortese, L., van de Sande, J., Lagos, C. P., et al. 2019, MNRAS, 485, 2656

Cui, W., Power, C., Knebe, A., et al. 2016, MNRAS, 458, 4052

Cui, W., Knebe, A., Yepes, G., et al. 2018, MNRAS, 480, 2898

De Luca, F., De Petris, M., Yepes, G., et al. 2021, MNRAS, 504, 5383

Doroshkevich, A. G. 1970, Astrofizika, 6, 581

Dressler, A. 1980, ApJ, 236, 351

Elahi, P. J., Knebe, A., Pearce, F. R., et al. 2016, MNRAS, 458, 1096

Errani, R., \& Peñarrubia, J. 2020, MNRAS, 491, 4591

Fall, S. M. 1983, in Internal Kinematics and Dynamics of Galaxies, ed. E. Athanassoula, IAU Symp., 100, 391

Fall, S. M., \& Romanowsky, A. J. 2018, ApJ, 868, 133

Fujita, Y. 1998, ApJ, 509, 587

Fujii, M., Funato, Y., \& Makino, J. 2006, PASJ, 58, 743

Gill, S. P. D., Knebe, A., \& Gibson, B. K. 2004, MNRAS, 351, 399

Gunn, J. E., \& Gott, J. R., III 1972, ApJ, 176, 1

Haggar, R., Gray, M. E., Pearce, F. R., et al. 2020, MNRAS, 492, 6074

Hashimoto, Y., \& Oemler, A., Jr. 2000, ApJ, 530, 652

Hayashi, E., Navarro, J. F., Taylor, J. E., Stadel, J., \& Quinn, T. 2003, ApJ, 584, 541

Hunter, J. D. 2007, Comput. Sci. Eng., 9, 90

Jiang, I.-G., \& Binney, J. 2000, MNRAS, 314, 468

Klimentowski, J., Łokas, E. L., Knebe, A. E., et al. 2010, MNRAS, 402, 1899

Klypin, A., Yepes, G., Gottlöber, S., Prada, F., \& Heß, S. 2016, MNRAS, 457, 4340

Knebe, A., Power, C., Gill, S. P. D., \& Gibson, B. K. 2006, MNRAS, 368, 741

Knebe, A., Gámez-Marín, M., Pearce, F. R., et al. 2020, MNRAS, 495, 3002

Knollmann, S. R., \& Knebe, A. 2009, ApJS, 182, 608

Kuchner, U., Aragón-Salamanca, A., Pearce, F. R., et al. 2020, MNRAS, 494, 5473

Kuchner, U., Aragón-Salamanca, A., Rost, A., et al. 2021, MNRAS, 503, 2065

Lagos, C. D. P., Theuns, T., Stevens, A. R. H., et al. 2017, MNRAS, 464, 3850
Lagos, C. D. P., Stevens, A. R. H., Bower, R. G., et al. 2018a, MNRAS, 473, 4956

Lagos, C. D. P., Schaye, J., Bahé, Y., et al. 2018b, MNRAS, 476, 4327

Li, Q., Cui, W., Yang, X., et al. 2020, MNRAS, 495, 2930

Łokas, E. L. 2020, A\&A, 638, A133

Mazzarini, M., Just, A., Macciò, A. V., \& Moetazedian, R. 2020, A\&A, 636, A106

Miller, T. B., van den Bosch, F. C., Green, S. B., \& Ogiya, G. 2020, MNRAS, 495,4496

Moore, B., Katz, N., Lake, G., Dressler, A., \& Oemler, A. 1996, Nature, 379, 613

Moore, B., Lake, G., \& Katz, N. 1998, ApJ, 495, 139

Mostoghiu, R., Knebe, A., Cui, W., et al. 2019, MNRAS, 483, 3390

Mostoghiu, R., Arthur, J., Pearce, F. R., et al. 2021, MNRAS, 501, 5029

Muldrew, S. I., Pearce, F. R., \& Power, C. 2011, MNRAS, 410, 2617

Obreschkow, D., \& Glazebrook, K. 2014, ApJ, 784, 26

Onions, J., Knebe, A., Pearce, F. R., et al. 2012, MNRAS, 423, 1200

Park, C., Choi, Y.-Y., Vogeley, M. S., et al. 2007, ApJ, 658, 898

Peñarrubia, J., Navarro, J. F., \& McConnachie, A. W. 2008, ApJ, 673, 226

Peebles, P. J. E. 1969, ApJ, 155, 393

Planck Collaboration XIII. 2016, A\&A, 594, A13

Planelles, S., Fabjan, D., Borgani, S., et al. 2017, MNRAS, 467, 3827

Pontzen, A., Roškar, R., Stinson, G., \& Woods, R. 2013, Astrophysics Source Code Library [record ascl:1305.002]

Power, C., Elahi, P. J., Welker, C., et al. 2020, MNRAS, 491, 3923

Rasia, E., Borgani, S., Murante, G., et al. 2015, ApJ, 813, L17

Recchi, S. 2014, Adv. Astron., 2014, 750754

Romanowsky, A. J., \& Fall, S. M. 2012, ApJS, 203, 17

Rost, A., Kuchner, U., Welker, C., et al. 2021, MNRAS, 502, 714

Sales, L. V., Navarro, J. F., Schaye, J., et al. 2010, MNRAS, 409, 1541

Schulze, F., Remus, R.-S., Dolag, K., et al. 2018, MNRAS, 480, 4636

Sembolini, F., Elahi, P. J., Pearce, F. R., et al. 2016a, MNRAS, 459, 2973

Sembolini, F., Yepes, G., Pearce, F. R., et al. 2016b, MNRAS, 457, 4063

Smith, R., Davies, J. I., \& Nelson, A. H. 2010, MNRAS, 405, 1723

Smith, R., Sánchez-Janssen, R., Beasley, M. A., et al. 2015, MNRAS, 454, 2502

Smith, R., Choi, H., Lee, J., et al. 2016, ApJ, 833, 109

Teklu, A. F., Remus, R.-S., Dolag, K., et al. 2015, ApJ, 812, 29

Valtonen, M. J., Valtaoja, L., Sundelius, B., Donner, K. J., \& Byrd, G. G. 1990, Celest. Mech. Dyn. Astron., 48, 95

van den Bosch, F. C. 2017, MNRAS, 468, 885

van der Walt, S., Colbert, S. C., \& Varoquaux, G. 2011, Comput. Sci. Eng., 13, 22

Vega-Ferrero, J., Dana, J. M., Diego, J. M., et al. 2021, MNRAS, 500, 247

Virtanen, P., Gommers, R., Oliphant, T. E., et al. 2020, Nat. Meth., 17, 261

Wang, Y., Pearce, F. R., Knebe, A., et al. 2016, MNRAS, 459, 1554

Wang, Y., Pearce, F., Knebe, A., et al. 2018, ApJ, 868, 130

White, S. D. M. 1984, ApJ, 286, 38

White, S. D. M., \& Rees, M. J. 1978, MNRAS, 183, 341 